\title{
Development of a Finger Mounted Type Haptic Device Using a Plane Approximated to Tangent Plane
}

\author{
Makoto Yoda and Hiroki Imamura \\ Department of Information System Science, Graduate School of Engineering, Soka University, Tokyo, Japan
}

\begin{abstract}
In recent years, several researches of haptic devices have been conducted. By using conventional haptic devices, users can perceive touching an object, such as CG (computer graphics) by a force feedback. Since conventional haptic devices provide a force feedback from a single point on an object surface where users touched, users touch an object by point contact. However, conventional haptic devices cannot provide users with a sense such as humans touching an object with a finger pad because a finger pad does not touch an object by point contact but surface contact in reality. In this paper, we propose a novel haptic device. By using this haptic device, users can perceive the sense of the slope on a CG object surface when they put fingers on it without tracing. Moreover, users can perceive the sense of grabbing a CG object with finger pads. To perceive the sense of the slope on a CG object and to grab it, we mount the plane interface of the haptic device on each two finger pads. Each plane interface provides a finger pad with the sense of the slope approximated to a tangent plane of area where they are touching. In the evaluation experiments, the subjects in this experiment evaluated this haptic device. From the results, the subjects could perceive the sense of the slope on a CG object surface. In addition, they could perceive the sense of grabbing a CG object.
\end{abstract}

Key words: Haptic device, plane interface, surface contact, force feedback.

\section{Introduction}

In recent years, researches of human interface using AR (augmented reality) have been conducted. In order to touch CG objects that are drawn by AR, haptic devices have been developed. By using a haptic device, users can perceive touching CG objects by processing a force feedback. Therefore, haptic devices are expected to be used in applications such as a virtual surgery simulation system and a virtual experience system.

Examples of conventional haptic devices include Falcon, PHANToM and Dexmo. Falcon and PHANToM are classified into a grounded type. This type can provide users with accurate force feedback because its fulcrum is fixed on the Table. Dexmo is classified into a finger mounted type. This type can provide users with perception of grabbing CG objects easily. In addition, users can operate the device

Corresponding author: Hiroki Imamura, Assoc. Prof., research fields: haptic device, image recognition, computer graphics. without operating range limitation. These haptic devices have been developed as a point contact type haptic device. By using this type of haptic devices, users can perceive touching CG objects because they are provided with a force feedback from a single point on the CG object surface where they touched. In case of perception of an objects shape, from visual information, users perceive CG objects shape of visible part. As invisible part, users must perceive CG objects shape by touching the surface. Humans perceive an object shape from the direction of a force feedback. However, in a point contact, as shown in Fig. 1, it is difficult to perceive an object shape because the direction of a force feedback changes according to a direction of a finger. To perceive CG objects shape in point contact, users must trace the surface.

In order to perceive a CG object shape without tracing the surface, a force feedback must be provided to the normal direction on a surface where users touched. To provide a force feedback to the normal 
Fig. 1 Characteristic of point contact.
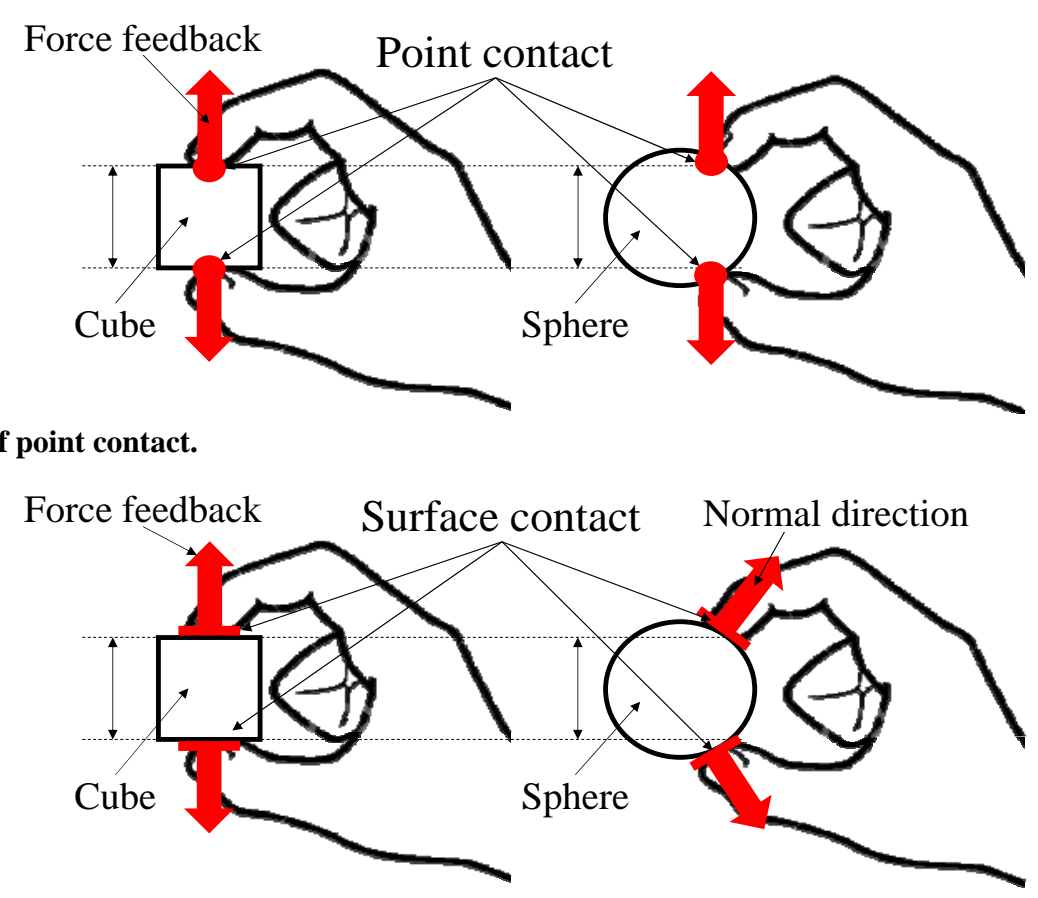

Fig. 2 Characteristic of surface contact.

direction, users touch an object by surface contact as shown in Fig. 2.

We focused on this characteristic. In addition, we have developed an HaAP (haptic device based on an approximated plane) [1] that is shown in Fig. 3 and Fig. 4, respectively. HaAP is a grounded type haptic device. Moreover, it is a surface contact type haptic device which has a plane interface as shown in Fig. 3.

As shown in Fig. 4, a plane interface provides a finger pad with a force feedback to normal direction on an object surface where users touched regardless of the direction of a finger. To provide a force feedback to the normal direction, a plane interface is approximated to tangent plane on a surface where users touched. Therefore, users can perceive an object shape with a finger pad without tracing a surface. In addition, in this device, users are limited to operate HaAP in range of the HaAP vertical mechanism within $10 \mathrm{~cm}$ as shown in Fig. 3.

In this paper, we propose a novel haptic device. This haptic device realizes three things. First, users are provided with a force feedback to normal direction by the plane interface approximated to the tangent plane on a CG object surface. Second, users can operate this haptic device without operating range limitation. Third, by using this haptic device, users can perceive grabbing a CG object. To realize that, we develop a haptic device having characteristics of a finger mounted type and a surface contact type.

\section{Our Approach}

Fig. 5 shows the outline of the proposed haptic

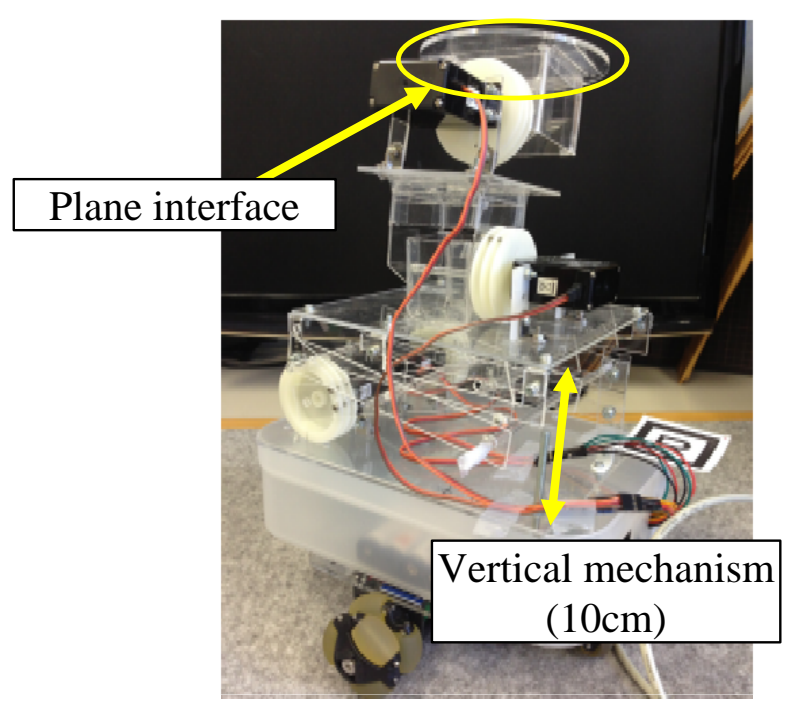

Fig. 3 Appearance of HaAP. 


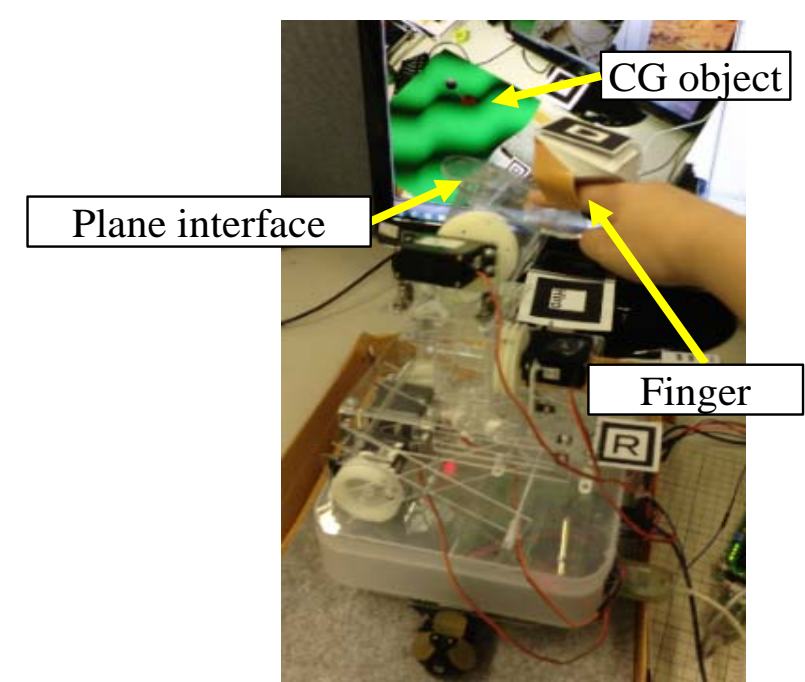

Fig. 4 HaAP in the operating state.

device in the initial state and in the operating state. This device uses a plane interface having four movable points. These four movable points operate up and down separately. In the initial state, the user is not touching a CG object. In the operating state, the user is touching a CG object. The plane interface provides a finger pad with a force feedback to the normal direction by four movable points operating up and down and being approximated to the tangent plane on the CG object surface where a finger pad touched.

\section{The Proposed System}

\subsection{Hardware Construction}

Fig. 8 shows the appearance of the proposed haptic

The plane interface is not providing a force feedback

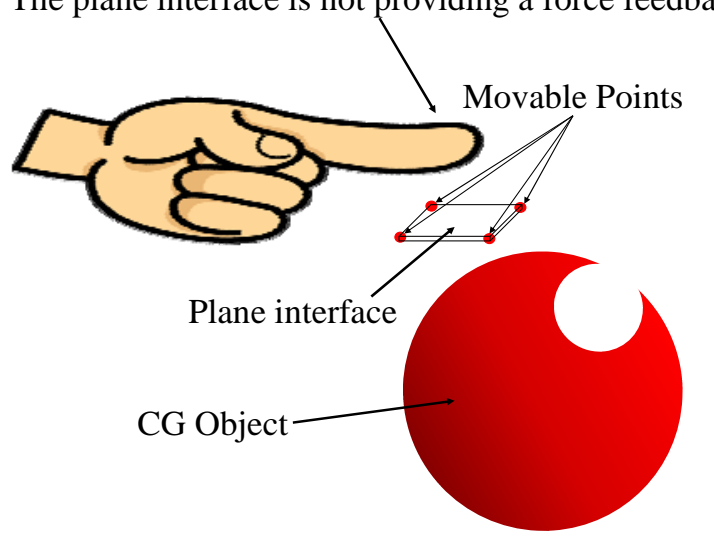

Initial State

Fig. 5 Outline of the proposed haptic device. device. This device is a glove type and composed of Arduino Uno that is shown in Fig. 6, four servo motors (GWS Servo PIC+F/BB/F) that is shown in Fig. 7, four springs, a plane interface and a marker for each finger. Eight motors are mounted in the back of the hand. To detect finger position and posture, markers are mounted in fingertips. In addition, each motor is connected to Arduino Uno for the index finger and for the thumb, respectively. Each Arduino Uno controls four motors in each finger. These motors pull up the movable points of the plane interface with wires. Each spring adheres to each movable point as shown in Fig. 9.

\subsection{System Overview}

Fig. 10 shows the system overview. This system consists of PC, Display, Web-camera, Reference marker and the proposed haptic device.

\subsection{The System Flowchart}

Fig. 11 shows the system flowchart. In the following section, we explain each process in the flowchart.

(1) Initialization of the Haptic Device

Arduino UNO controls motors to make springs natural length.

(2) Drawing a CG Object

Based on the reference marker, by using ARToolkit

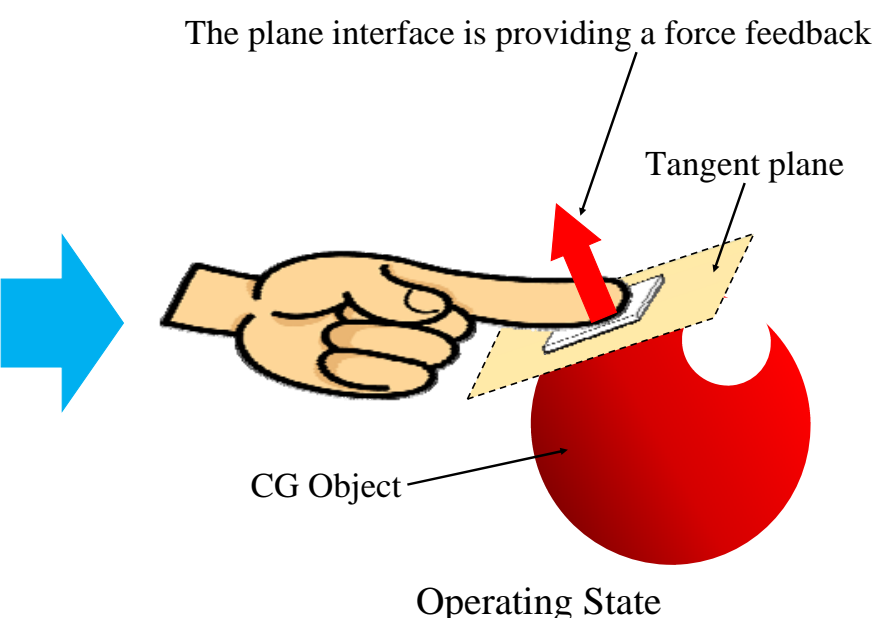

Operating State 


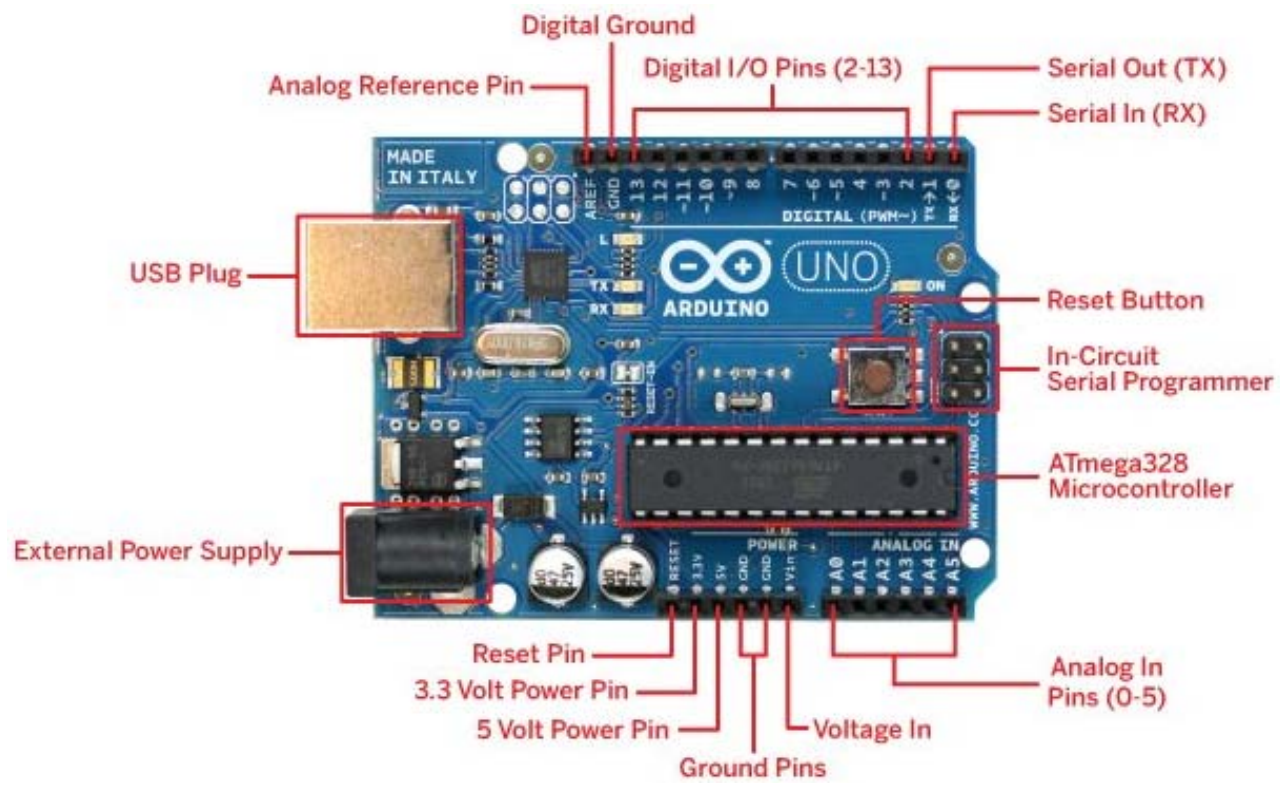

Fig. 6 Arduino UNO.

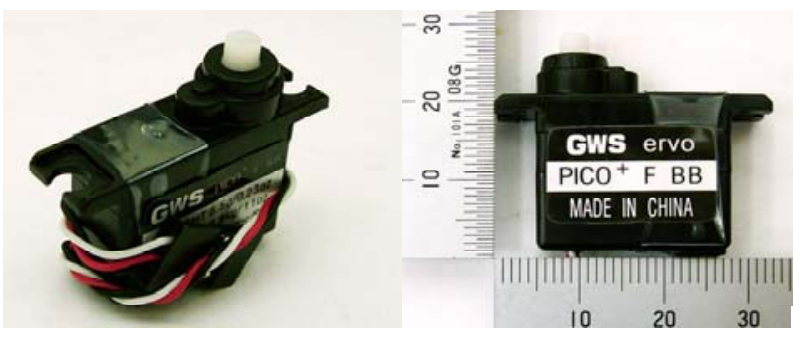

Fig. 7 Servo motors (GWS Servo PIC+F/BB/F).

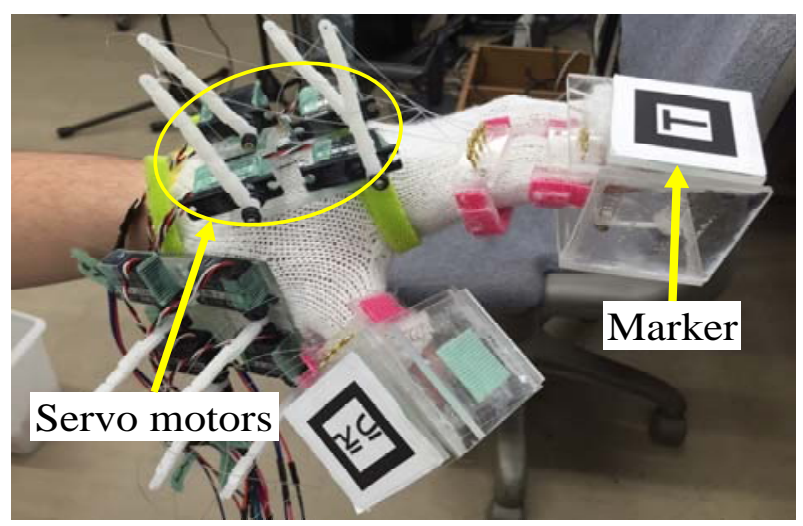

Fig. 8 Appearance of the proposed haptic device.

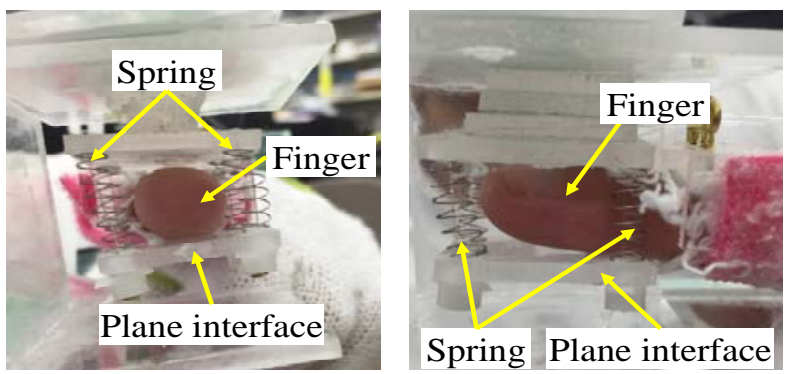

Fig. 9 The fingertip part of the proposed haptic device.

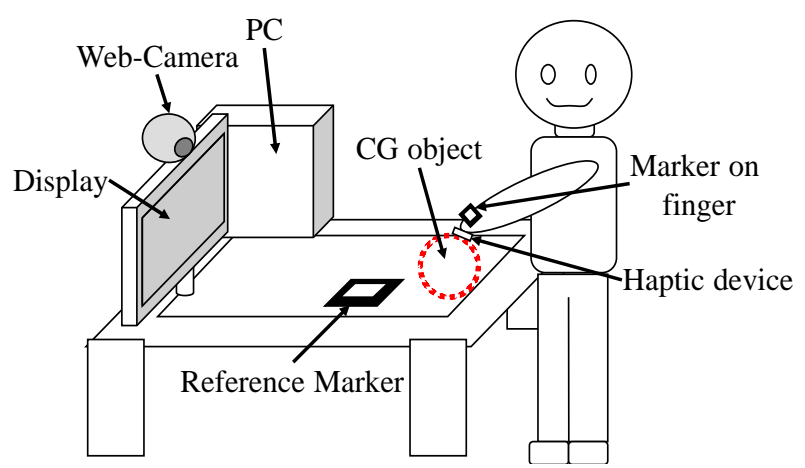

Fig. 10 System overview.

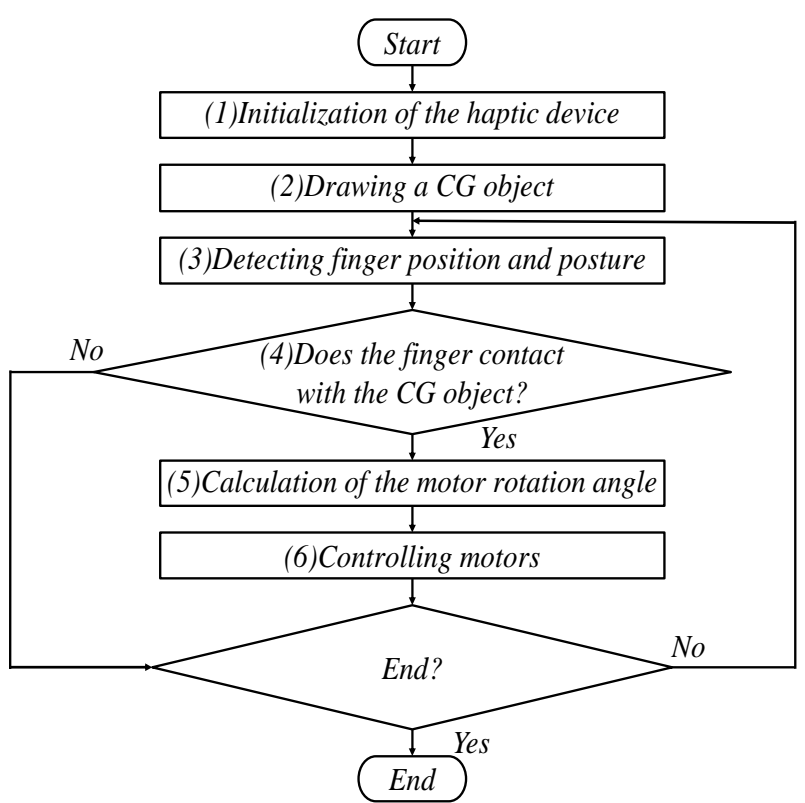

Fig. 11 Flowchart. 
[2], this system draws Sphere CG object or Sin-cos curve CG object that is shown in Figs. 12 and 13 respectively.

(3) Detecting Finger Position and Posture

Fig. 14 shows detecting the marker on each finger position and posture.

The system recognizes the reference marker and the marker on each finger from the image that is captured by the Web-camera. By using ARToolKit, the system obtains position $(X, Y, Z)$ of the marker on each finger from position $(0,0,0)$ of the reference marker. We defined this position as a finger position. In addition, by using ARToolKit, the system also calculates $F_{r}$ that denotes the roll angle and $F_{p}$ that denotes the pitch angle of the marker on each finger.

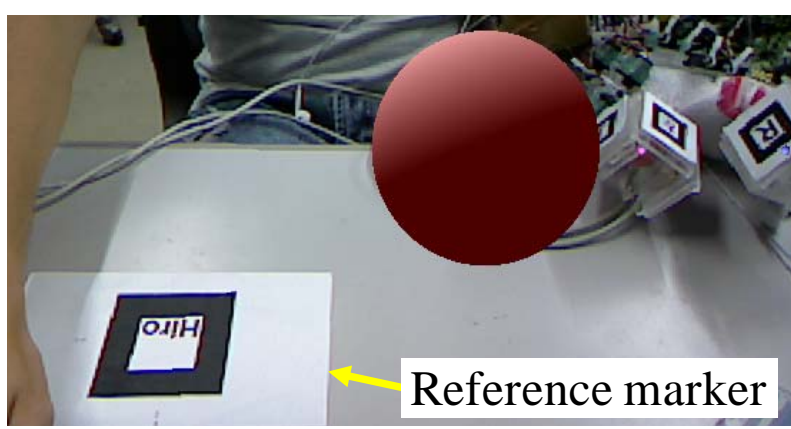

Fig. 12 Sphere CG object.

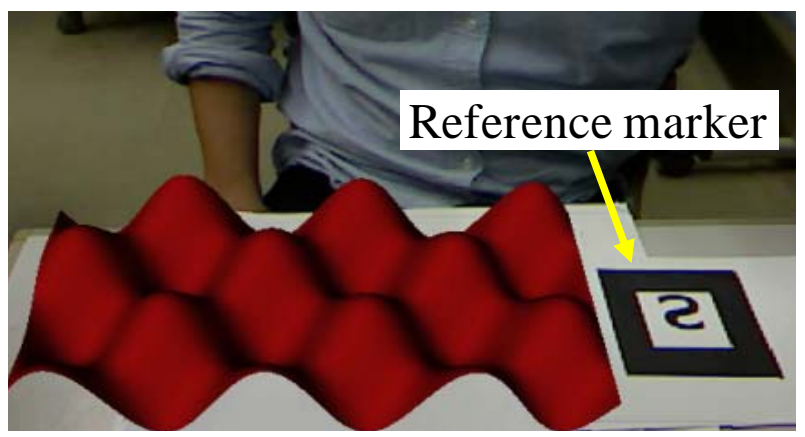

Fig. 13 Sin-cos curve CG object.

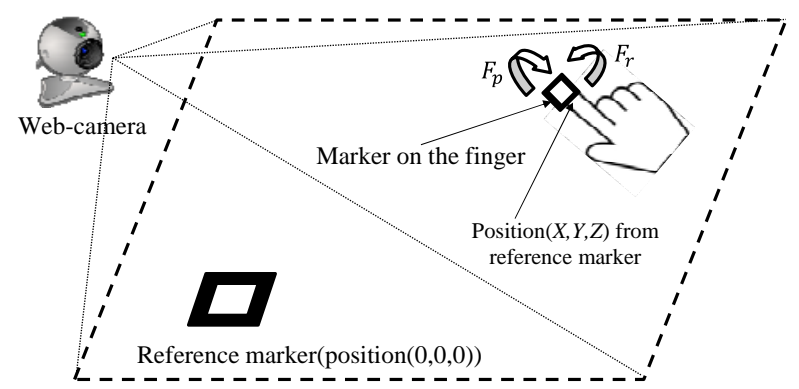

Fig. 14 Detecting the finger position and posture.

\section{(4) Judgement of Contact}

In case of Sphere CG object, as shown in Fig. 15, when the length between the finger position and the center of Sphere CG object is within radius of Sphere CG object, the system judges contact.

In case of Sin-cos curve CG object, the system calculates Z-coordinate on Sin-cos curve CG object. The following is the equation of Sin-cos curve.

$$
A \sin X \cos Y=Z
$$

where $A$ is amplitude and $X, Y$ and $Z$ are $\mathrm{X}, \mathrm{Y}$ and $Z$-coordinate on Sin-cos curve CG object. By substituting $\mathrm{X}$ and $\mathrm{Y}$-coordinate of the finger position for this equation, the system obtains $Z$. As shown in Fig. 16, when Z-coordinate of the finger position is under $Z$, the system judges contact.

(5) Calculation of the Motor Rotation Angle

The system calculates the normal vector on touching point to calculate the tangent plane slope.

In case of Sphere CG object, the vector from the center of Sphere CG object to the touching point is

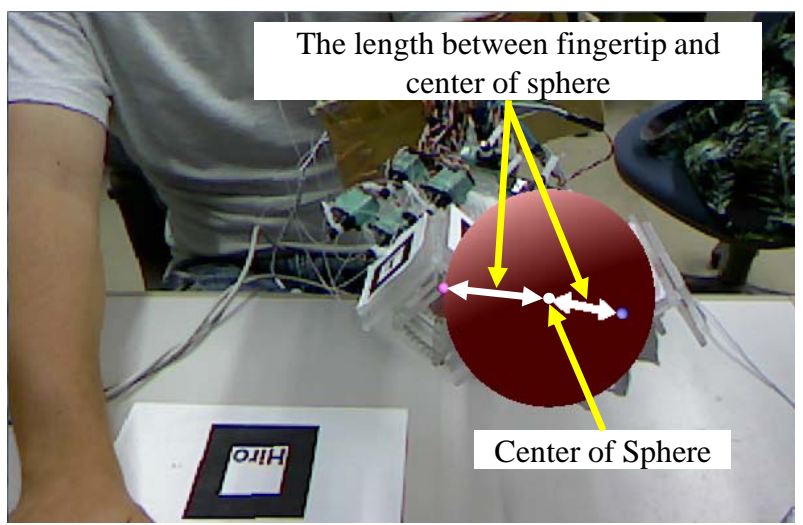

Fig. 15 Touching Sphere CG object.

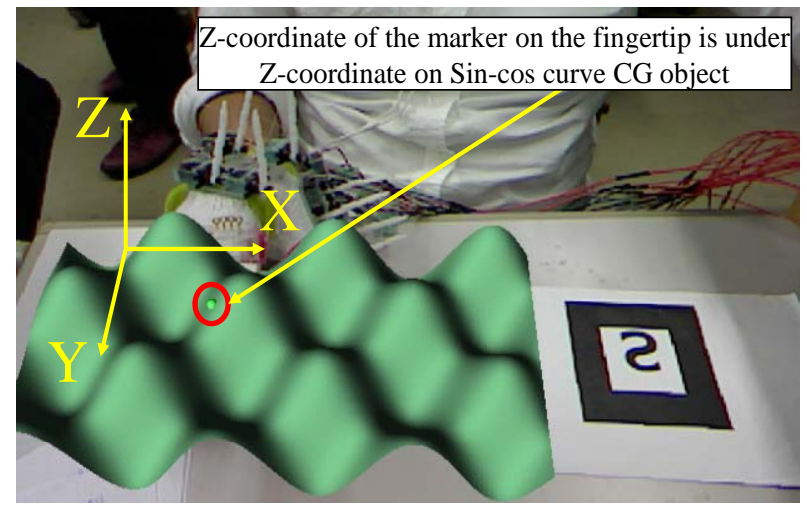

Fig. 16 Touching Sin-cos curve CG object. 
defined as the normal vector on the touching point.

In case of Sin-cos curve CG object, Sin-cos curve CG object is composed of many planes. Fig. 17 shows calculation of the normal vector on the touching point. The system uses the equation of vector product

$$
\boldsymbol{N}=\boldsymbol{A} \times \boldsymbol{B}
$$

where $\boldsymbol{A}$ and $\boldsymbol{B}$ are the vectors from the touching point to other points on the plane that includes the contact point. The system obtains the normal vector from right hand screw rule.

From the normal vector

$$
\boldsymbol{N}=\left(\begin{array}{c}
N_{x} \\
N_{y} \\
N_{z}
\end{array}\right)
$$

the system calculates

$$
\theta_{x}=\cos ^{-1} \frac{\left|N_{x}\right|}{\sqrt{N_{x}{ }^{2}+N_{z}^{2}}}
$$

that denotes the angle between $\mathrm{x}$-axis and the normal vector in $\mathrm{X}-\mathrm{Z}$ plane and

$$
\theta_{y}=\cos ^{-1} \frac{\left|N_{y}\right|}{\sqrt{N_{y}^{2}+N_{z}^{2}}}
$$

that denotes the angle between $y$-axis and the normal vector in Y-Z plane that is shown in Fig. 18.

Fig. 19 shows the calculation of the tangent plane slope on touching point in $\mathrm{X}-\mathrm{Z}$ plane and $\mathrm{Y}-\mathrm{Z}$ plane. Since the normal vector is vertical to the tangent plane, the system uses

$$
\begin{aligned}
& T_{p}=90^{\circ}-\theta_{y} \\
T_{r}= & 90^{\circ}-\theta_{x}
\end{aligned}
$$

to obtain the roll and pitch angle of the tangent plane. Using Eqs. (6) and (7), the system obtains $T_{r}$ that denotes the roll angle of tangent plane and $T_{p}$ that denotes the pitch angle of the tangent plane.

Using Eqs. (8) and (9), the system calculates the difference between the angle of the marker on the finger and that of the tangent plane.

$$
\begin{aligned}
& D_{p}=\left|T_{p}-F_{p}\right| \\
& D_{r}=\left|T_{r}-F_{r}\right|
\end{aligned}
$$

This difference is the plane interface slope that is shown in Figs. 20 and 21. $F_{p}$ and $F_{r}$ are shown in Fig. 14. Fig. 20 shows $D_{p}$ that denotes the difference

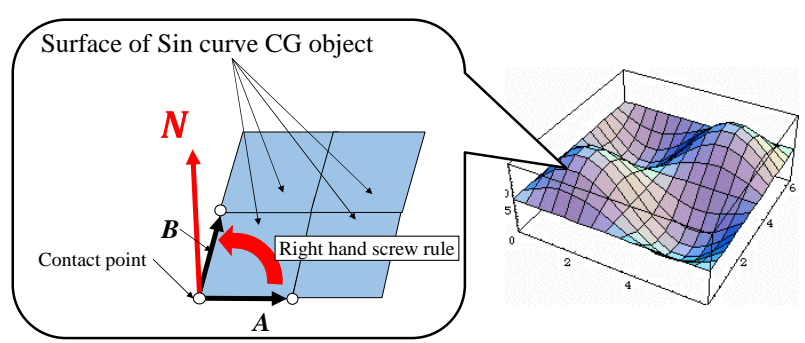

Fig. 17 Calculation of the normal vector in case of Sin-cos curve CG object.

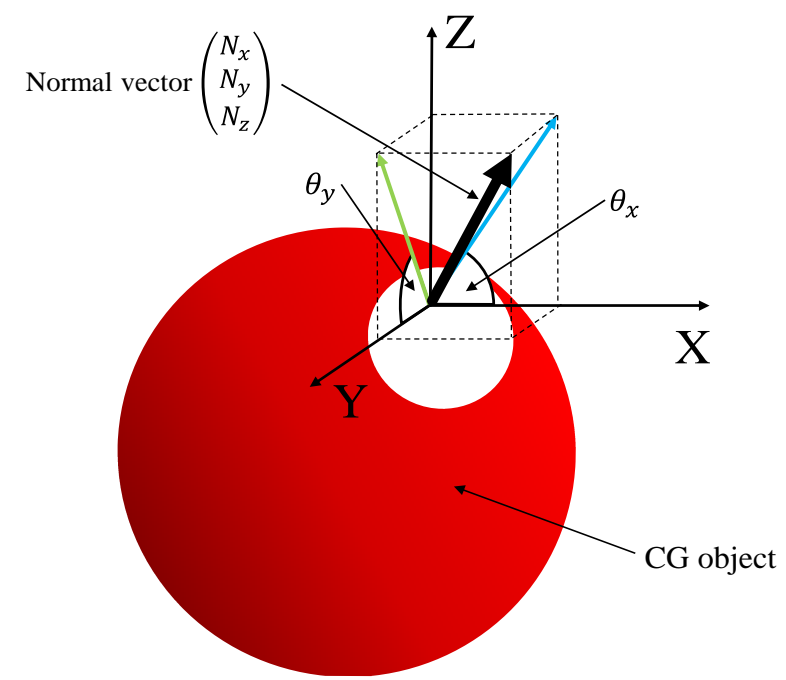

Fig. 18 Calculation of $\theta_{x}$ and $\theta_{y}$.
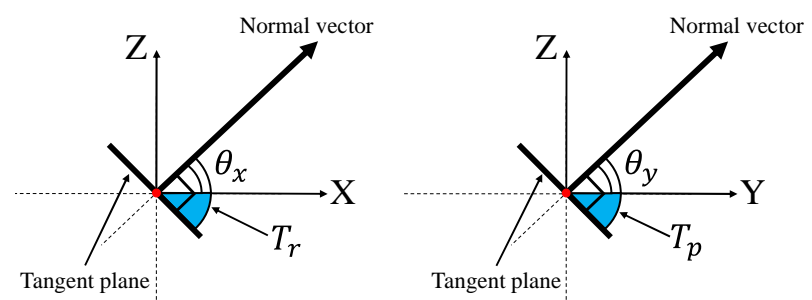

Fig. 19 Calculation of tangent plane slope.

between $F_{p}$ and $T_{p}$. Fig. 21 shows $D_{r}$ that denotes the difference between $F_{r}$ and $T_{r}$.

The system calculates the operation length of movable point by using the plane interface slope. Fig. 22 shows the state transition of a plane interface. A plane interface operates as shown in this figure.

Fig. 23 shows two lengths (L1 and L2). These two lengths are defined

$$
\begin{array}{r}
L 1=\frac{A}{2} \sin \left(\left|D_{p}\right|\right)+\frac{A}{2} \sin \left(\left|D_{r}\right|\right) \\
L 2=\left|\frac{A}{2} \sin \left(\left|D_{p}\right|\right)-\frac{A}{2} \sin \left(\left|D_{r}\right|\right)\right|
\end{array}
$$




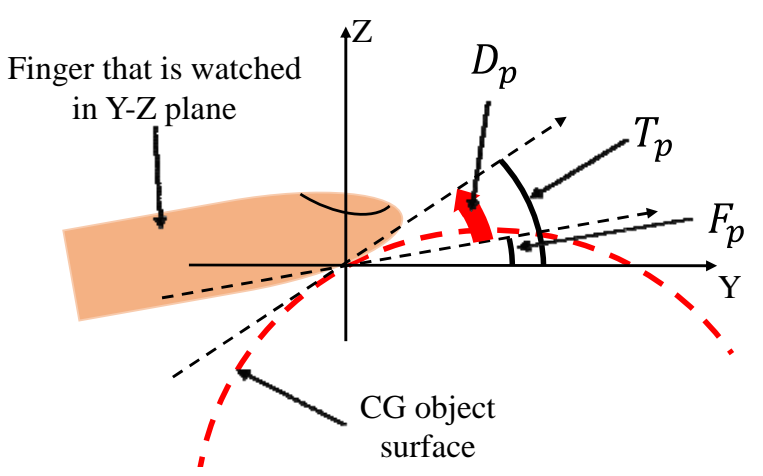

Fig. 20 Calculation of the difference of the pitch angle.

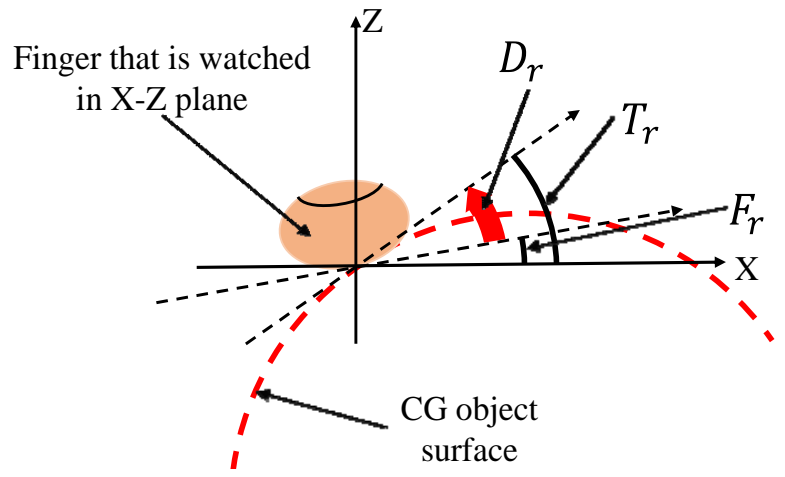

Fig. 21 Calculation of the difference of the roll angle.
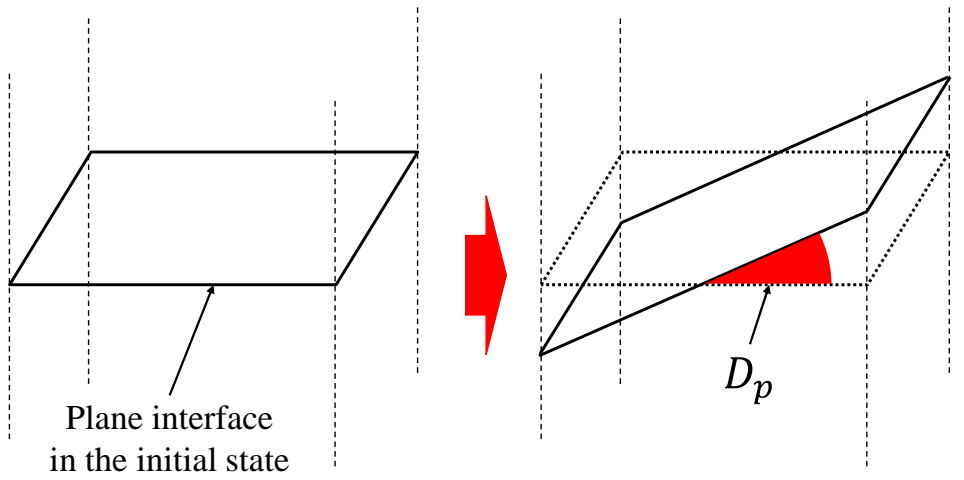

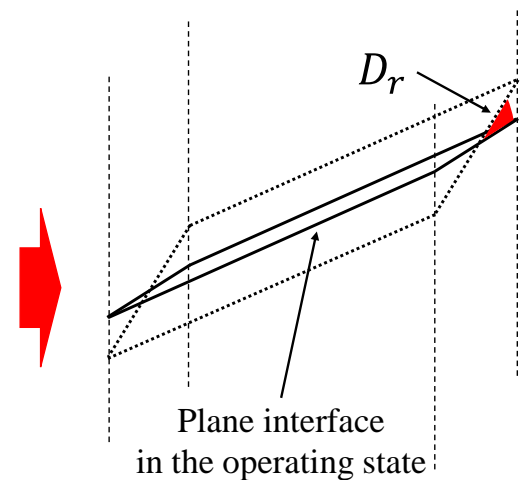

Fig. 22 The state transition of a plane interface.

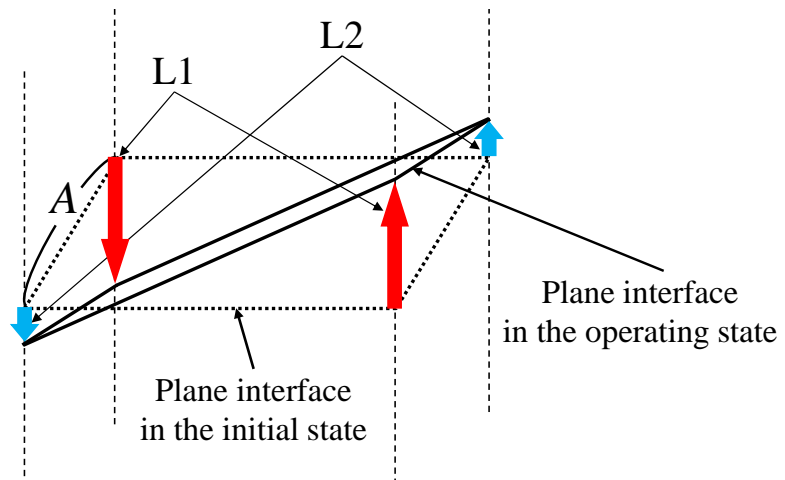

Fig. 23 Calculation of the operation length of movable points.

as the operation length of movable points. Where $A$ is the length of one side on a plane interface. Using Eqs. (10) and (11), the system calculates these lengths.

Fig. 24 shows the angle of motor rotation. The system uses Eqs. (12) and (13)

$$
\begin{aligned}
& A 1=2 \sin ^{-1}\left(\frac{L 1}{2 R}\right), \\
& A 2=2 \sin ^{-1}\left(\frac{L 2}{2 R}\right)
\end{aligned}
$$
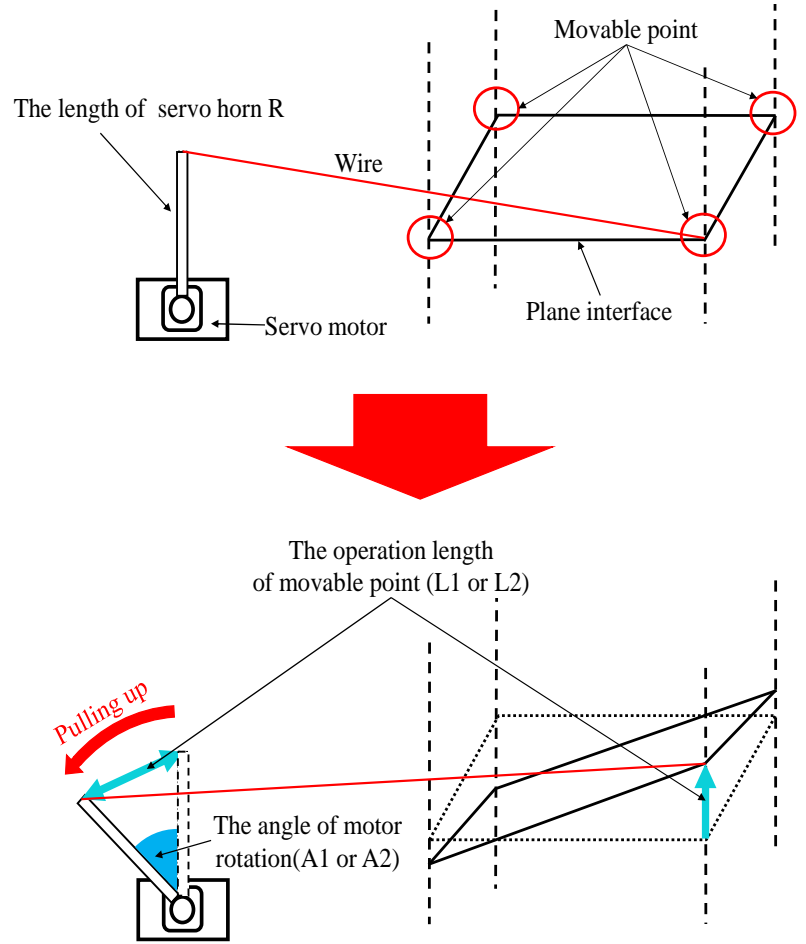

Fig. 24 Calculation of the motor rotation angle. 
to calculate the angle of motor rotation, where $\mathrm{R}$ denotes the length of servo horn. A1 and A2 are the amount of controlling motors. The system sends this amount to each Arduino Uno by serial communication.

(6) Controlling the Motors

Arduino Uno receives the amount of controlling motors and controls motors. Motors pull up each movable point with wires. Figs. 25 and 26 show providing a finger with a force feedback when users touched Sphere CG object in Y-Z plane and X-Z plane respectively. Figs. 27 and 28 show providing a finger with a force feedback when users touched Sin-cos curve CG object in $\mathrm{Y}-\mathrm{Z}$ plane and $\mathrm{X}-\mathrm{Z}$ plane respectively.

\section{Evaluation Experiments}

\subsection{Overview of Experiments}

We had evaluation experiments for the proposed haptic device. Sin-cos curve CG object is used in order to evaluate whether users can perceive the sense of the slope on the CG object surface or not. In addition, a Sphere CG object is used in order to evaluate whether users can perceive grabbing a CG object or not. Eleven subjects used the proposed haptic device. After that, they evaluated following items with a 5-grade score:

In case of Sin-cos curve CG object,

- When you touched the CG object with one finger, you perceived the sense of the slope on the CG object surface at once (Item 1).

- When you touched and traced the surface of CG object with one finger, you perceived the sense of the asperity on the CG object surface (Item 2).

In case of Sphere CG object,

- When you touched the CG object with one finger, you perceived the sense of the slope on a spherical surface (Item 1).

- When you touched and traced the surface of the CG object with one finger, you perceived the shape of a spherical surface (Item 2).

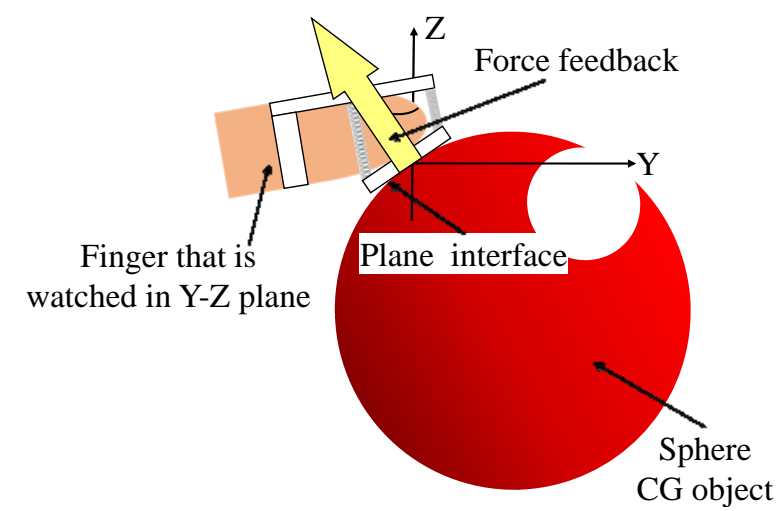

Fig. 25 Touching sphere CG object in Y-Z plane.

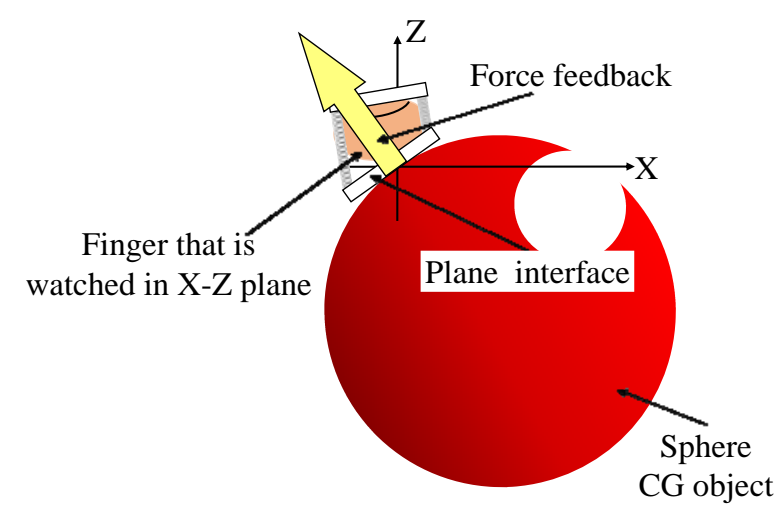

Fig. 26 Touching sphere CG object in X-Z plane.

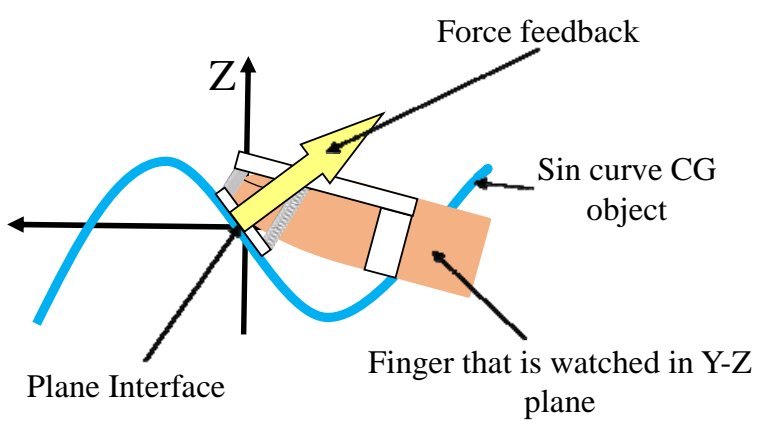

Fig. 27 Touching Sin-cos curve CG object in Y-Z plane.

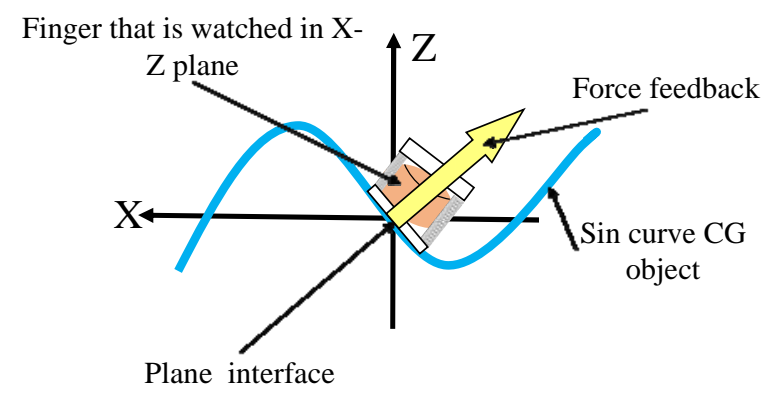

Fig. 28 Touching Sin-cos curve CG object in X-Z plane. 
Table 1 Results of Sin-cos curve CG object.

\begin{tabular}{lll}
\hline & \multicolumn{2}{c}{ Sin-cos curve CG object } \\
\hline Item & Average score & A standard deviation \\
\hline 1 & 4.64 & 0.64 \\
2 & 4.55 & 0.50 \\
\hline
\end{tabular}

Table 2 Results of Sphere CG object.

\begin{tabular}{lll}
\hline & \multicolumn{2}{c}{ Sphere CG object } \\
\hline Item & Average score & A standard deviation \\
\hline 1 & 4.09 & 0.90 \\
2 & 4.00 & 0.85 \\
3 & 4.18 & 0.94 \\
4 & 4.27 & 0.96 \\
\hline
\end{tabular}

- When you touched the CG object with two fingers, you perceived the sense of touching the CG object (Item 3).

- When you touched the CG object with two fingers, you perceived the sense of grabbing the CG object (Item 4).

Evaluation values are from 1 to 5 (1: "Strongly disagree,” 2: “Disagree”, 3: “Neutral”, 4: “Agree”, 5: "Strongly agree”).

\subsection{Discussion}

Table 1 shows the results of Sin-cos curve CG object. Table 2 shows results of Sphere CG object. Each result shows the average score and the standard deviation.

From these results, in Item 1 of Tables 1 and 2, we see that users perceived the sense of the slope on CG object surface without tracing the surface. In Item 2 of Tables 1 and 2, the results show that users perceived the asperity by tracing the surface. In addition, in Items 3 and 4 of Table 2, we see that users perceived grabbing the CG object. Therefore, we consider that the proposed haptic device can provide users with perception of CG object surface shape by providing the sense of the slope and the asperity of CG object surface. Moreover, we consider that users can perceive grabbing a CG object by using the proposed haptic device. In addition, from Tables 1 and 2, we see that the average score in Table 1 is higher than that in Table 2. Since the surface of Sin-cos curve CG object is more complex than that of Sphere CG object, the accuracy of the proposed haptic device is improved when a CG object has complex surface.

\section{Conclusion and Future Works}

In this paper, we proposed a novel haptic device. The proposed haptic device has a plane interface. The plane interface provides a finger with a force feedback to normal direction by being approximated to tangent plane on a CG object surface where users touched. In addition, to perceive grabbing a CG object, the proposed haptic device is designed as a finger mounted type. After evaluation experiments, we see that the proposed haptic device can provide users with perception of the CG object surface shape and perception of grabbing the CG object. However, we consider that users cannot grasp the sense of the distance between finger and a CG object easily. To solve the issue, we improve the proposed haptic device to grasp the sense of the distance more easily by using HMD (head mounted display). In the future, we will improve the operability of the proposed haptic device by lightening the device. In addition, by using Leap Motion, we will improve the accuracy of detecting finger position and posture.

\section{References}

[1] Kawazoe, A., Ikeshiro, K., and Imamura, H. 2013. "A Haptic Device Based on an Approximate Plane HaAP.” Presented at the ACM SIGGRAPH Asia 2013 Posters, Hong Kong, CD-ROM.

[2] Kato, H. 2002. ARToolKit: Library for Vision-based Augmented Reality. Technical report of IEICE, PRMU. 